\title{
Efficacy of Nd-YAG laser iridotomies in acute angle closure glaucoma
}

\author{
R H GRAY, J HOARE NAIRNE, AND W H R AYLIFFE \\ From Bristol Eye Hospital, Lower Maudlin Street, Bristol BS1 2 LX
}

SUmmaRY Between September 1984 and April 1987 a total of 150 patients underwent Nd-YAG laser iridotomy procedures at the Bristol Eye Hospital. Forty of these patients $(27 \%)$ were treated for acute angle closure glaucoma (AACG), and three of them (7\%) suffered recurrent AACG. Sixteen patients who had been treated for AACG have been challenged by a pharmacological provocative test introduced by Mapstone, with a total of 23 eyes being tested. A negative result was obtained in all cases. Provocative testing is recommended to help identify those at risk of recurrent AACG.

Laser iridotomy has gained widespread favour over surgical iridectomy because of factors such as avoidance of retrobulbar anaesthesia, maintenance of the anterior chamber, absence of risk of infective endophthalmitis, and application as an outpatient procedure. Nd-YAG is considered preferable over argon because of lower total energy requirements,' greater ease of iris penetration independent of iris colour, ${ }^{2}$ and a lower incidence of iridotomy closure. ${ }^{34}$ The long term safety of argon iridotomy compares favourably with that of surgery, ${ }^{5}$ and initial concern about cataract formation with YAG iridotomy has not been confirmed. ${ }^{67}$

This study was undertaken to review the short term results of YAG laser iridotomies for AACG since its introduction in Bristol in 1984, and to assess the efficacy of iridotomies by provocative testing of a selected group of patients.

\section{Material and methods}

For the purposes of this study acute angle closure glaucoma (AACG) was defined as a red, painful eye, with elevated intraocular pressure in the presence of a closed anterior chamber angle. The case notes of all patients undergoing YAG iridotomies during September 1984 to April 1987 were reviewed. Sixteen patients were recalled for provocative testing, which was performed according to a technique developed by Mapstone. ${ }^{8}$ Patients were selected on the basis of previous YAG iridotomy and no subse-

Correspondence to Mr R H Gray. Fowlers Bench. Upper Burwardsley. Tattenhall. Cheshire CH3 9PF. quent ocular hypotensive medications being required. One drop each of $10 \%$ phenylephrine and $2 \%$ pilocarpine was instilled into both eyes at 3minute intervals for nine minutes after baseline intraocular pressures had been recorded by Goldmann applantation tonometry. Pressures were checked again at 1 hour, at which time the test was considered positive if a rise of $8 \mathrm{mmHg}$ or greater had occurred. If negative at this stage, further drops were instilled if pupil size had not reached $3 \mathrm{~mm}$ diameter; otherwise pressures were checked again at 2 hours. The test was considered negative if the pressure had not risen by $8 \mathrm{mmHg}$ by 2 hours. (Pupil diameter was measured by adjusting a focused slit beam on the Haag-Streit biomicroscope.)

\section{Results}

In the period studied 150 patients underwent one or more YAG laser iridotomies. Of these patients $40(27 \%)$ were treated for acute angle closure glaucoma, $13(9 \%)$ for subacute angle closure glaucoma, $44(29 \%)$ for chronic glaucoma in which the anterior chamber angles were felt to be pathologically narrow, $17(11 \%)$ for iris bombé (most often due to pseudophakia or posterior synechiae), 22 $(15 \%)$ for prophylaxis on narrow angles, $3(2 \%)$ for phacogenic glaucoma, and one after silicone oil insertion. Information could not be obtained on $\mathbf{1 0}$ patients $(7 \%)$.

Of the 40 patients in the AACG group one has been lost to follow-up and one died in hospital, while the remaining 38 have been reviewed at intervals in 
Table 1 Surgical procedures required (acute angle closure glaucoma)

\begin{tabular}{lcc}
\hline Treatment & $\begin{array}{l}\text { Acute eyes } \\
(n=38)\end{array}$ & $\begin{array}{l}\text { Fellow eyes } \\
(n=34)\end{array}$ \\
\hline Single iridotomy & $16(42 \%)$ & $28(82 \%)$ \\
Two iridotomies* & $10(26 \%)$ & $4(12 \%)$ \\
Three iridotomies* & $2(6 \%)$ & 0 \\
Iridotomy(ies)+PI & $4(10 \%)$ & 1 \\
Iridotomy(ies)+trabeculectomy & $6(16 \%)$ & 1 \\
\hline
\end{tabular}

*At separate sittings. $\mathrm{PI}=$ Peripheral iridectomy.

the clinic and form the subject of this study. All patients had developed unilateral AACG, so 38 eyes with acute glaucoma were studied. Of the 38 fellow eyes, one eye had already undergone a trabeculectomy, one was rubeotic, and one was pseudophakic. Thirty-four fellow eyes were treated prophylactically. The average age of the patients was 74 , and females predominated (75\%). All patients studied were Caucasian.

A Lasag Neodynium YAG Series 2 Microruptor with CGI1 contact lens was used throughout. In $\mathrm{Q}$ switch and multimode, typical power settings were 5-10 $\mathrm{mJ}$, and the number of pulses varied between 8 and 350 . Of the 103 iridotomies carried out in total on the 38 patients studied, 20 were carried out by consultants, the remainder by junior medical staff, with a total of 15 different operators being involved (Table 1).

All but three of the patients studied were admitted to hospital for medical treatment prior to laser therapy. This involved the use of intravenous and oral acetozolamide (Diamox), and $4 \%$ pilocarpine eye drops, both of which were continued at least until the iridotomy. The interval between admission and laser varied from less than 12 hours to five days, with the highest IOP recorded at the time of iridotomy being $35 \mathrm{mmHg}$. Corneal oedema was recorded in five eyes with acute glaucoma at the time of iridotomy, and four of these required further YAG procedures. Bleeding from the iridotomy site was recorded in six eyes with acute glaucoma and one fellow eye, but in no case was the procedure abandoned. $70 \%$ of patients had a bilateral procedure carried out at the first sitting, with the remaining patients having the fellow eye treated at a later date. The average number of nights spent in hospital was five.

In nearly half of the eyes with acute onset a single iridotomy was successful, while the remaining eyes were treated again because the original iridotomy was judged to be too small or imperforate (Table 1). Four $(10 \%)$ acute eyes went on to surgical peripheral iridectomy: on two eyes because the iridotomies were obviously imperforate, on one eye after recurrent
Table 2 Results of provocative testing*

\begin{tabular}{llllll}
\hline & \multicolumn{2}{l}{ Zero hour } & & \multicolumn{2}{l}{2 Hours } \\
\cline { 2 - 3 } \cline { 5 - 6 } & $P D$ & IOP & & $P D$ & IOP \\
\hline Acute eyes $(\mathrm{n}=15)$ & $2.75 \mathrm{~mm}$ & $15.4 \mathrm{mmHg}$ & & $3.85 \mathrm{~mm}$ & $14.3 \mathrm{mmHg}$ \\
Fellow eyes $(\mathrm{n}=14)$ & $2.47 \mathrm{~mm}$ & $15.3 \mathrm{mmHg}$ & & $3.48 \mathrm{~mm}$ & $13.3 \mathrm{mmHg}$ \\
\hline
\end{tabular}

*Average values. $\mathrm{PD}=$ Pupil diameter. $\mathrm{IOP}=$ Intraocular pressure.

AACG, and on the remaining eye because of persistently raised intraocular pressure despite a clinically patent iridotomy. Six (15\%) eyes with acute glaucoma went on to trabeculectomy, in all cases because of persistently raised IOPs while on topical medications and with a clinically patent iridotomy.

Three eyes (7.5\%) suffered recurrent AACG, at a maximum post-YAG interval of six weeks, and all had previously undergone more than one YAG iridotomy. The first eye was still receiving pilocarpine when the patient presented again, and the iridotomy looked patent. A trabeculectomy was carried out. The iridotomy of the second eye did not look patent, and a further iridotomy was performed. Iris bleeding occurred, and this iridotomy was also imperforate. Following a peripheral iridectomy the IOP became normal without any medications. The original iridotomy of the third eye was judged small at an early follow-up appointment, and the patient soon developed recurrent AACG. After a further YAG iridotomy the IOP was normal on no treatment.

Sixteen patients attended for provocative testing, with 16 acute and 15 fellow eyes being tested (Table 2). One patient did not stay for the required two hours, but was negative in both eyes at 1 hour. Three patients were still using pilocarpine, but all achieved the required pupil dilatation. In the 15 eyes with acute glaucoma the average pupil diameter rose from $2.75 \mathrm{~mm}$ to $3.85 \mathrm{~mm}$ at 2 hours, and the average IOP was $15.4 \mathrm{mmHg}$ initially and $14.3 \mathrm{mmHg}$ after two hours. In the 14 fellow eyes the average pupil diameter rose from $2.47 \mathrm{~mm}$ to $3.48 \mathrm{~mm}$ after two hours, and the average IOP changed from 15.3 $\mathrm{mmHg}$ to $13.3 \mathrm{mmHg}$. In none of the 29 eyes tested did the IOP rise more than $2 \mathrm{mmHg}$. In two eyes the maximum pupil diameter was $2.75 \mathrm{~mm}$; all the other eyes achieved a pupil diameter of $3 \mathrm{~mm}$ or greater.

\section{Discussion}

Laser iridotomy has become an attractive alternative to surgical peripheral iridectomy in the treatment of AACG. One advantage of Nd-YAG over argon has been the smaller number of repeat iridotomies needed. ${ }^{4}$ However, $48 \%$ of the eyes with acute glaucoma and $18 \%$ of fellow eyes required retreat- 
ment, well above the $9 \%$ quoted by Schwartz et al., ${ }^{2}$ but their group of 200 eyes included only 42 with AACG. Our figures indicate that eyes with acute onset are more difficult to treat, probably owing to factors such as corneal transparency and thickness, and iris oedema. In addition junior staff carried out most of our procedures and were probably on a 'learning curve'. ${ }^{2}$

The increased incidence of iris bleeding in acute eyes (16\% compared to $3 \%$ in fellow eyes) is consistent with their engorged iris vasculature, yet the number is small compared with one reported study of $45 \% .{ }^{4}$ These eyes, however, were monitored for five minutes at the slit-lamp after the iridotomy, which was not the case in our study.

Analysis of the available clinical data did not reveal any factors that would be predictive of the number of laser procedures required. When those requiring only one procedure were compared with those needing more than one procedure, the average length of acute attack was found to be 2.6 days in the former and 1.82 in the latter. Similarly, presenting IOPs were almost identical in the two groups. Although iris colour was not analysed, previous studies have not shown darker irides to be more difficult to penetrate with the YAG laser. ${ }^{13}$

Nineteen eyes with acute glaucoma have required no further treatment after YAG iridotomy(ies). For this group the average IOP at presentation and the average length of acute attack were $57 \mathrm{mmHg}$ and 3.3 days respectively. This compares with values of 55 $\mathrm{mmHg}$ and 2.8 days for the remaining 19 eyes with acute glaucoma requiring further medical or surgical treatment. These figures do not indicate that length of acute attack increases the risk of chronic angle closure glaucoma developing, and lend support to the policy of delaying iridotomy until IOP has been reduced.

Recurrent AACG does not appear to be a significant risk after surgical peripheral iridectomy, ${ }^{910}$ but was an important complication after YAG iridotomy in our series. The three eyes that developed recurrent AACG had undergone more than iridotomy at the time of their first attack, but when they presented again it was clear in two that the iridotomy was imperforate. A further YAG iridotomy was carried out on one, and a peripheral iridectomy on the other, and neither of these eyes required further treatment. The third eye had a plateau iris configuration, and the patient presented again despite a clinically patent iridotomy. A trabeculectomy was carried out.

Closure of Nd-YAG iridotomies was not observed in one reported study ${ }^{3}$ of 38 patients with acute or chronic angle closure glaucoma over a mean period of 15 months, patency being verified in these patients by direct inspection of the iridotomy site. A report of a larger series ${ }^{2}$ of $200 \mathrm{Nd}-\mathrm{YAG}$ iridotomies describes two closures during a mean follow-up of 9.3 months, these occurring in patients with pre-existing chronic uveitis. It is likely that in one of our three recurrences the iridotomy was imperforate from the outset, but it is possible that in one patient the iridotomy closed later. This is well recognised with argon laser iridotomies.' The third eye developed recurrent AACG in the presence of a clinically patent iridotomy, an observation consistent with the diagnosis of plateau iris made at that time.

Clinical assessment of iridotomy patency may be made in a number of ways. Direct observation of the crystalline lens or vitreous through the iridotomy is certainly more reliable than iris retroillumination in this respect. The appearance of a plume of iris pigment issuing from the iridotomy site is a good indicator that the posterior iris pigment layer has been breached, but this phenomenon does not always occur during a successful iridotomy. Resolution of iris bombé, with deepening of the peripheral anterior chamber, should also occur but may be difficult to detect during the iridotomy procedure. Quigley emphasises that provocative testing should be performed on all patients who have had laser iridotomies after pupil block glaucoma, and he performed provocative testing as part of a follow-up on patients having argon iridotomies."

The optimum size of a YAG laser iridotomy is not yet known, but gauging a minimum iridotomy size may be part of the learning curve mentioned earlier, going some way to explaining the recurrences in our patients.

Mapstone, using the technique described above, tested a group of 119 fellow eyes similar to ours ${ }^{12}$ and found that $55 \%$ gave positive results after two hours. When further provocative measures were used and the test period was extended to five hours, this figure rose to $70 \%$. During a mean follow-up of three years only one patient with a negative test result developed AACG. A negative result does not of course prove iridotomy patency, but a positive result indicates an inadequate iridotomy and the need for further treatment.

We consider that Nd-YAG iridotomies are a safe and effective treatment for AACG, but there is a small and significant risk of recurrence. Routine clinical methods may not identify those at risk, and therefore we advocate the routine use of Mapstone's test to help identify this group. We suggest that it be incorporated into the early follow-up of patients as soon as ocular hypotensive medications have been discontinued.

We thank the consultant staff at Bristol Eye Hospital for permission to report on their patients. 


\section{References}

1 Klapper RM. Q switched NdYAG laser iridotomy. Ophthalmology 1984; 91: 1017-21.

2 Schwartz LW, Moster MR, Spaeth GL, Wilson RP, Poryzees EM. NdYAG laser iridectomies in glaucoma associated with closed or occludable angles. Am J Ophthalmol 1986; 102: 41-4.

3 Moster ME, Schwartz LW, Spaeth GL, Wilson RP, McAllister JA, Poryzees EM. Laser iridectomy, a controlled study comparing argon and NdYAG. Ophthalmology 1986; 93: $20-4$.

4 Robin AL, Pollack IP. A comparison of NdYAG and argon laser iridotomies. Ophthalmology 1984; 91: 1011-6.

5 Robin AL, Pollack IP. Argon laser peripheral iridotomies in the treatment of primary angle closure glaucoma. A long term follow-up. Arch Ophthalmol 1982; 100: 919-23.

6 Tomey KF, Traverso CE, Shammes IV. NdYAG laser iridotomy in the treatment and prevention of angle closure glaucoma. A review of 373 eyes. Arch Ophthalmol 1987; 105: 476-81.

7 Tomey KF. Efficacy and safety of NdYAG laser iridotomy in angle closure glaucoma. Glaucoma 1985; 7: 107-9.

8 Mapstone R. The fellow eye. Br J Ophthalmol 1981; 65: 410-3.

9 Floman N, Berson D, Landau L. Peripheral iridectomy in closed angle glaucoma. Late complications. Br J Ophthalmol 1977; 61: $101-4$.

10 Krupin T, Mitchell KB, Johnson MF, Becker B. The long term effects of iridectomy for primary acute angle closure glaucoma. Am J Ophthalmol 1978: 86: 506-9.

11 Quigley HA. Long term follow-up of laser iridotomy. Ophthalmology 1981; 88: 218-24.

12 Mapstone $\mathrm{R}$. Provocative tests in closed angle glaucoma. $\mathrm{Br} \mathrm{J}$ Ophthalmol 1976: 60: 115-9.

Accepted for publication 17 March 1988. 